\title{
CORRESPONDENCE
}

\section{Primary pulmonary hypertension}

Anonymous .

Asthma mortality: comparison between $\mathrm{New}$ Zealand and England

I W B Grant, FRCP; P T White, MRCGP ..........

Hazard of potassium chloride solution C Hawkins, FRCP ............................

Continuous ambulatory peritoneal dialysis D G Oreopoulos, FRCP(C); A G Morgan, FRCP, and R P Burden, FRCP; S F Beardsworth, $M R C P$, and $R$ Ahmad, MB; P Burton, MB, and J Walls, FRCP...............................
Prognosis for infants born at 23 to 28 weeks'

L W Doyle, FRACP, and W H Kitchen,

FRACP .........................................

Computer aided diagnosis of acute abdominal

pain

P Driscoll, FRCS, and C Vincent, MPHIL; F T

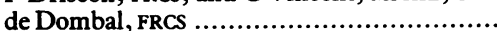

Prolonged pregnancy

J F R Barrett, $M B$, and others

Prevalence of $\mathrm{HBsAg}$ in UK population

Elizabeth $\mathrm{H}$ Boxall, MRCPATH, and $\mathrm{T} H$

Flewett, FRCPATH; M A Vickers, MRCP, and

54
Improvement in bronchial hyperresponsiveness in patients with moderate asthma after treatment with a hypnotic technique

55 DR Graham, MRCP, and J Lewis, MD ........... Academic boycotts of South Africans A Seedat, MD ..................................

Points Assaying creatinine (Anne Cruickshank and A Shenkin); Non-mydriatic fundus photography in detection of diabetic retinopathy (R E J Ryder); Generalised granuloma annulare and hypothyroidism (A Y Finlay and others); A better way to detect growth failure (E C Coles); Chlamydial infections in Papua 57 New Guinea (W A Hart) ........................

Because we receive many more letters than we have room to publish we may shorten those that we do publish to allow readers as wide a selection as possible. In particular, when we receive several letters on the same topic we reserve the right to abridge individual letters. Our usual policy is to reserve our correspondence columns for letters commenting on issues discussed recently (within six weeks) in the BMF.

Letters critical of a paper may be sent to the authors of the paper so that their reply may appear in the same issue.

We may also forward letters that we decide not to publish to the authors of the paper on which they comment.

Letters should not exceed 400 words and should be typed double spaced and signed by all authors, who should include their main degree.

\section{Primary pulmonary hypertension}

SIR,-Dr Tim Higenbottam's leading article (6 December, $p$ 1456) is an impressive account of state of the art scientific medicine. My practice has a patient who has received treatment for this condition and is now back in the community, complete with central venous line through which she is receiving prostacyclin. It has improved her quality of life, but my partners and I have had anguished discussions about the moral dilemmas raised by her case.

We are prescribing the prostacyclin Flolan (Wellcome), which costs $£ 103$ per ampoule (according to our local pharmacist), and she is receiving two ampoules every 12 hours. The cost to the community budget of improving this one patient's quality of life is therefore $£ 150000$ a year, plus the cost of all the infusing equipment. This is double the average GP's expenditure on drugs for his or

her entire list. I have telephoned my family practitioner committee and regional medical officer, who have informed me that I am perfectly free to prescribe this drug if I feel it is "clinically justified." Having been told by the hospital that she was being discharged to the community on this drug, how can her GP then refuse to prescribe it if he does not feel it to be clinically justified because of its great expense, and the lack of evidence that it will change the prognosis of the patient's condition?

It is clear that this form of treatment can be afforded. The question remains whether it should be afforded in a cost limited health service. Is this where the priority for limited funds should lie when renal physicians are being asked to select patients for dialysis because of limited resources?

ANONYMOUS

\section{Asthma mortality: comparison between New Zealand and England}

SIR,-It must have been tempting for Dr M R Sears and colleagues (22 November, $p$ 1342), who have previously published a nationwide study of asthma deaths in New Zealand, ' to compare their findings with those of a similar study conducted in two regions of England. ${ }^{2}$ The comparison has, however, two major flaws.

Firstly, and common to both studies, the age group (15-64 years) studied is more liable to diagnostic erro than, say, a 5-34 age group, since in older patients the cause of death may be chronic obstructive airways disease rather than asthma. That error may well have differed in size between the two studies and, if so, might have influenced the reported difference in "asthma mortality" (table I).

Secondly, the New Zealand study conducted from August 1981 to July 1983 was compared with one conducted in England two to three years previously. Apart from the inherent disadvantage of using what is in effect a historical control group, there is the more specific objection that a single radical difference between the two studies on therapeutic policy, such as the use of bronchodilator nebulisers in the home (common in New Zealand in 1981-3 but virtually non-existent in England in 1979), may have influenced other aspects of patient care. These could have included the prescription of other drugs, such as oral corticosteroids, the patient's perception of when it is essential to call a doctor, and perhaps even the genera practitioner's perception of the need to respond immediately to such a call so that admission to hospital, if indicated, is not delayed.

No evidence is available to indicate whether the use of bronchodilator nebulisers in the home contributed to the recent increase in the number of deaths from asthma in New Zealand. To test such a hypothesis one would have to identify all patients for whom that treatment was available in 1981-3 (there were at leas 6000 in $1982^{3}$ ) and compare their death rate with that of a group of matched controls, who might comprise, for example, patients whose asthma was severe enough to require nebulised bronchodilators in hospital or at a health centre but who did not subsequently acquire a nebuliser for domiciliary use. The collection of data for such a comparison would be formidable but not impossible. If the controls had a lower mortality that could incriminate nebulisers as a possible cause of death, whereas the opposite finding would suggest that they might help to prevent a fatal outcome. Without that type of information the comparison made in the article of the drug treatment given in the two studies is not particularly useful. I am equally unimpressed by the comparative assessments of patient compliance and "preventable deaths" between the two studies, since these rely on the insecure processes of memory recall and subjective judgment.

An earlier article from New Zealand' further illustrates the importance, when making comparisons with England and Wales, of excluding the Maori and Pacific Island Polynesian population from asthma mortality statistics. These two ethnic groups in the $1981-3$ study accounted for $48 \%$ of the deaths from asthma in those aged 5-34, although they constituted only about $15 \%$ of the population. If the same is broadly true of earlier and later years the peak total death rate of $4 \cdot 1$ per 100000 in 1979 would represent a death rate for caucasians of $2 \cdot 4$ (3.5 times the figure for England and Wales) and that of $2 \cdot 2$ in 1983 a caucasian death rate of 1.3 (only 1.5 times the figure for England and Wales). There are indications, as yet unconfirmed, of a further fall towards the 1985 English figure of 0.88 per 100000 for the same age group.

Assuming that the pattern I have identified for the 5-34 age group is based on valid figures (and the authors will no doubt challenge me if they are wrong) the following conclusions emerge.

(1) In caucasians mortality from asthma increased from 1.3 times the figure for England and Wales in 1975 to 3.5 times in 1979. It fell back to 1.5 times the England and Wales figure by 1983 and may now be lower still.

(2) This, the second "epidemic" of asthma deaths in New Zealand, waxed and waned over about five years (1976 to 1981 or 1982), much as did the first epidemic between 1965 and 1970 . Between the two epidemics the total death rate (including all ethnic groups) remained much higher in New Zealand than in England and Wales, but that could be explained by the very high mortality in Maoris and Pacific Island Polynesians (8.6 per 100000 for the 5-34 age group in $1981-3, v 1 \cdot 5$ in caucasians).

(3) Two rises and falls in asthma mortality, each lasting about fives years, occurring over 20 years are unlikely to be caused by "natural" factors such as changes in the incidence or severity of asthma, in environmental allergen exposure, or in the prevalence of viral infections. Changes in patient care related to the introduction and supervision of new forms of drug treatment would seem to be of much greater relevance. 
Although the second epidemic of asthma deaths in New Zealand started in 1976-7, that epidemic may now have subsided. Asthma mortality among caucasians in New Zealand may even no longer be significantly greater than mortality in England and Wales, assuming that comparisons restricted to the 5-34 age group achieve an acceptable degree of diagnostic accuracy. If these observations-or predictions-were to be correct the gloomy 1981-3 contrast between asthma mortality in New Zealand and England drawn by the authors could safely be consigned to history, provided that the valuable lessons which emerged from the two asthma deaths studies are diligently learned, and never forgotten, by all doctors and patients.

IAN W B GRANT

Kirknewton,

West Lothian EH27 8EA

1 Sears MR, Rea HH, Beaglehole R, et al. Asthma mortality in New Zealand: a two year national study. NZ Med f 1985;98: 271-5.

2 British Thoracic Association. Death from asthma in two regions of England. Thorax 1982;285:1251-5.

3 O'Donnell TV. Home nebulisers for asthmatics. NZ Med $\mathcal{f}$ 1982;95:637.

SIR,-Dr M R Sears and colleagues (22 November, p 1342) suggest that a GP with 1200 patients aged 15-64 should expect to see one death from asthma in this age group among New Zealand caucasians about every 20 years (mortality $4 \cdot 2$ / 100000 or 0.05 deaths per 1200 patients per year). The figures in the British Thoracic Association's report in 1982 suggest that a GP with 1200 patients aged 15-64 should expect to see one death from asthma in this age group among people of unspecified race in England and Wales every 50 years (mortality $1.84 / 100000$ or 0.02 deaths per 1200 patients per year). This latter rate arises from the 1500 deaths caused by asthma in England and Wales each year, a rate of $1 / 1000$ to $1 / 3000$ asthmatics each year, depending on whose assessment of the prevalence of asthma you accept. ${ }^{2}$

These figures indicate how difficult it is to extrapolate any assessment of general practice care from the numbers involved. None the less, much criticism of GPs' routine care of patients with asthma has been derived from the abstract of the British Thoracic Association's report, although careful reading of the report itself shows that the blame for unavoidable deaths was placed mainly on the delay, for whatever reason, between the onset of the fatal attack and the provision of appropriate treatment.

Dr Sears and colleagues have avoided placing the emphasis on delay in their detailed and enlightening discussion, although if we were to take together cases where delay was a possible cause and cases where failure to use corticosteroids in the fatal attack was a possible cause the analysis of avoidable deaths would show delay in obtaining appropriate treatment as the major preventable factor.

The question which these two studies have tried to address is: Was death the result of inadequate long term management or of inadequate treatment of the acute attack? That the answer is largely the latter of these two possibilities should not be a surprise. There is virtually no evidence to support the theory that the modern drug treatment of asthma should substantially reduce either the morbidity or mortality of chronic asthma, although the drugs are without doubt effective in treating acute asthma. No studies of the long term effect on morbidity or mortality among asthmatics in the general population has been carried out in respect of inhaled or oral steroids or inhaled cromoglycate. All three are significantly better than placebo in improving lung function in selected populations of chronic asthmatics, but the effect on morbidity has been dubious to say the least. Comment about the appropriateness of treatment before the onset of the fatal attack, therefore, seems of limited relevance.

Since adrenaline might have been as effective in treating acute asthma as we now find $\beta$ agonists nebulised with oxygen to be, perhaps these mortality studies show why it is that mortality from asthma has not changed in 20 if not 100 years. Could it be that both severely dyspnoeic asthmatics and their physicians have been taking the adage "festina lente" too literally for too long.

PATRICK T WhITE

London SE27 0EH

1 British Thoracic Association. Deaths from asthma in two regions of England. Br Med $\mathcal{F}$ 1982;285:1251-5

Gregg I. Epidemiological aspects. In: Clark TJH, Godfrey S, eds. Asthma. London: Chapman and Hall, 1983:242-84.

\section{Hazard of potassium chloride solution}

SIR,-Deaths from potassium instead of sodium chloride continue to occur but tend to be recorded in coroners' courts rather than medical journals. The latest was the same tragic story': a junior doctor injected the wrong ampoule into a 3 week old baby's intravenous drip, whereupon the heart beat dropped from 140 to 20 beats/min and then stopped.

My interest in this was aroused during a discussion with the secretary of the Medical Defence Union when writing a book (Mishap or Malpractice?) to celebrate its centenary. A visit to our pharmacy confirmed that the ampoules are identical in size, shape, lettering, and colour of solution; it surprised me that no steps had been taken by manufacturers or others to solve the problem by colour coding or changing the shape of the ampoules.

My pharmaceutical colleagues took the matter up, and Mr J E H Baker, pharmaceutical officer of Coventry Health Authority, designed an ampoule that cannot possibly be mistaken, for it has a fixed plastic cap over its neck and is manufactured by Antigen Ltd. It is used throughout the West Midlands region and has been approved by anaesthetists, nursing authorities, and others-and the old ampoules have been withdrawn by the pharmacists. The extra cost is trivial. The doctor should still read the label but it provides an added safeguard when dealing with an emergency or when a potassium chloride ampoule has inadvertently been put in the box of sodium chloride ampoules-like a seat belt even though the driver knows the highway code by heart.

\section{Clifford Hawkins}

Postgraduate Centre,

Queen Elizabeth Hospital,

1 Anonymous. Doctor injected baby with deadly solution. Guardian 1986 Nov 29:4 (col 6).

\section{Continuous ambulatory peritoneal dialysis}

SIR,-Drs A G Morgan and R P Burden described their experience with "long term" continuous ambulatory peritoneal dialysis (CAPD) (11 October, p 935). Their three year actuarial patient survival was $49 \%$ and technique survival $41 \%$. They also observed that patients who were transferred from CAPD to haemodialysis had a one year survival of only $64 \%$ and commented that "change in treatment is likely to introduce new hazards." This experience led them to recommend CAPD primarily for young patients awaiting a kidney transplant and to treat elderly patients by haemodialysis.

The last conclusion does not make sense since when discussing the treatment of elderly patients they say "in many cases we can treat [older patients] just as well with haemodialysis." Indeed, with regard to the elderly, this is the experience of Canadian centres (Posen G, Canadian Renal Failure Registry, personal communication)-that is, patient and technique survival of those aged over 65 is the same (or slightly better) with peritoneal dialysis as with haemodialysis. Since we both agree that the results of haemodialysis and CAPD are the same in the elderly why do Drs Morgan and Burden prefer haemodialysis when CAPD offers the advantages of treatment outside hospital and the freedom of home dialysis?

Although Drs Morgan and Burden provide no data, their paper gives the impression that their results with haemodialysis are better than those with CAPD. Can they provide data which allow comparison of haemodialysis and CAPD in patients of comparable age, underlying disease, and pre-existing cardiovascular disease? Such studies as have been done have shown that the results of CAPD (corrected for these three factors) were as good if not better than those obtained with hospital haemodialysis.

Of the deaths in their population a large proportion (eight out of 23) were due to peritonitis and an additional seven from other infections. Furthermore, 17 of the 33 patients undergoing CAPD who were moved to haemodialysis were transferred because of recurrent peritonitis. This high rate of infection probably explains their findings. Many readers would like to know how their infection rate compares with that of other experienced centres, most of which now have an incidence of about one episode every year, and whether they have dedicated enough nurses to their CAPD population; the ratio in well established centres is one nurse per 12-14 patients. As set out in the paper by Dr A Heaton and others (11 October, p 938), few studies, if any, find peritonitis and other sepsis to be the major cause of death among patients undergoing CAPD whereas recurrent peritonitis may still be the main cause of CAPD failure in some studies, though not in others. In a recent analysis of 126 patients who were in our CAPD programme from January 1983 to June 1984 we had 19 deaths and 15 failures; of the latter, nine were converted to haemodialysis and six to intermittent peritoneal dialysis. The main causes of death were cardiovascular or cerebrovascular. ${ }^{2}$ Only one patient died from peritonitis and two from other sepsis. Only three of the 15 failures were due to peritonitis.

I suspect that most of the authors' deaths on haemodialysis were early deaths related to complications of CAPD such as peritonitis. In most registries deaths within four to six weeks of transfer to haemodialysis are still considered to be CAPD deaths. An analysis of the cause of death and time after transfer among their patients would be revealing.

I believe that if CAPD is going to be successful it should be performed in centres that will pay as much attention to detail, training, and staffing levels for CAPD as they do for haemodialysis.

Peritoneal Dialysis Unit,

Toronto Western Hospital,

Toronto, Ontario, Canada MST 2S8

1 Hutchinson A, Harvey C. Survival with different forms of dialysis treatment. American Society of Artificial Internal Organs Journal 1985;8:13.

2 Mitrwalli A, Wu G, Schilling H, Oreopoulos DG. Complications. In: Khanna R, Nolph KD, Prowant B, Twardowski ZJ, Oreopoulos DG, eds. Patients underoing CAPD. Advances in CAPD. Toronto: Peritoneal Dialysis Bulletin, 1985:127.

AUTHORS' REPLY - We are sorry that Dr Oreopoulos seems upset that we questioned the wisdom of offering continuous ambulatory peritoneal dialysis (CAPD) as first choice of treatment to all patients with end stage renal failure. A statistical comparison between CAPD and haemodialysis was not the purpose of our paper and we do not have comparable groups of patients to allow this.

Our suggestion for the need for selectivity was 